\title{
Proceeding Paper \\ Overlooked Ionic Phenomena Affecting the Electrical Conductivity of Liquid Crystals ${ }^{\dagger}$
}

\author{
David Webb and Yuriy Garbovskiy *(D)
}

check for updates

Citation: Webb, D.; Garbovskiy, Y. Overlooked Ionic Phenomena

Affecting the Electrical Conductivity of Liquid Crystals. Eng. Proc. 2021, 11, 1. https://doi.org/10.3390/ ASEC2021-11141

Academic Editor: Nicholas Sarlis

Published: 15 October 2021

Publisher's Note: MDPI stays neutral with regard to jurisdictional claims in published maps and institutional affiliations.

Copyright: (c) 2021 by the authors. Licensee MDPI, Basel, Switzerland. This article is an open access article distributed under the terms and conditions of the Creative Commons Attribution (CC BY) license (https:// creativecommons.org/licenses/by/ $4.0 /)$.
Department of Physics and Engineering Physics, Central Connecticut State University, New Britain, CT 06050, USA; davidwebb@my.ccsu.edu

* Correspondence: ygarbovskiy@ccsu.edu

+ Presented at the 2nd International Electronic Conference on Applied Sciences, 15-31 October 2021; Available online: https://asec2021.sciforum.net/.

\begin{abstract}
Liquid crystal devices, such as displays, various tunable optical components, and sensors, are becoming increasingly ubiquitous. Basic physical properties of liquid crystal materials can be controlled by external physical fields, thus making liquid crystal devices dynamically reconfigurable. The tunability of liquid crystals offers exciting opportunities for the development of new applications, including advanced electronic and photonic devices, by merging the concepts of flat optics, tunable metasurfaces, nanoplasmonics, and soft matter biophotonics. As a rule, the tunability of liquid crystals is achieved by applying an electric field. This field reorients liquid crystals and changes their physical properties. Ions, typically present in liquid crystals in minute quantities, can alter the reorientation of liquid crystals through the well-known screening effect. Because the electrical conductivity of thermotropic liquid crystals is normally caused by ions, an understanding of ion generation processes in liquid crystals is of utmost importance to existing and emerging technologies relying on such materials. That is why measuring of electrical conductivity of liquid crystals is a standard part of their material characterization. Measuring the electrical conductivity of liquid crystals is a very delicate process. In this paper, we discuss overlooked ionic phenomena caused by interactions of ions with substrates of the liquid crystal cells. These interactions affect the measured values of the DC electrical conductivity of liquid crystals and make them dependent on the cell thickness.
\end{abstract}

Keywords: liquid crystals; liquid crystal devices; ions; ion generation; electrical conductivity

\section{Introduction}

Liquid crystals continue to be at the heart of modern technologies. They include display devices, such as ubiquitous liquid crystal displays (LCD) and miniature liquid crystal on silicon (LCoS) displays for virtual and augmented reality [1]. Active optical components made of liquid crystals can be found in numerous polarization and phase control devices, including liquid crystal variable retarders [2,3], tunable elements of biomedical equipment (dynamic lenses, optical filters for hyperspectral imaging, etc.) [4,5], spatial light modulators, and diffractive optical elements [6,7]. Reconfigurable components of plasmonic [8] and meta-devices [9] also take advantage of the tunability of liquid crystal materials. Switchable liquid crystal light shutters [10-12] and smart windows [13,14] are becoming increasingly ubiquitous. Last but not least, liquid crystals are excellent materials for the fabrication of reconfigurable microwave devices, including phase shifters, tunable antennas, filters, and resonators, to name just a few [15].

As a rule, the tunability of the aforementioned devices is achieved by exploiting the electric-field induced reorientation of liquid crystal materials [7]. This reorientation can be altered by the electric field screening effect caused by ions normally present in liquid crystals in minute quantities $[16,17]$. In the case of display devices, ions in liquid 
crystals can lead to many undesirable effects, including image flickering, image sticking, reduced voltage holding ratio, and overall slow response $[16,17]$. There are also liquid crystal applications relying on ions. For example, liquid crystal shutters [10-12] and smart windows $[13,14]$ are receiving increasing attention these days.

The importance of ions in liquid crystal materials has been recognized since the early 1960s $[16,17]$. Since that time, numerous reports have been published aimed at broadening our understanding of mechanisms of ion generation in liquid crystal materials ([16-18] and references therein).

Typically, information about ions in liquid crystals is obtained by performing electrical measurements [19-22]. Such measurements utilize sandwich-like liquid crystal cells [19-22]. The obtained experimental results are used to evaluate the value of the DC electrical conductivity, ion mobility, and their bulk concentration [19-22]. In the majority of the reports, electrical measurements are performed at only a single value of cell thickness [23]. At the same time, a very limited number of measurements carried out using several cells with different thicknesses reveal an important experimental fact that the measured electrical parameters of liquid crystals, in general, can depend on the cell gap $[20,21,24,25]$. Unfortunately, only a very limited set of experimental data is available. An analysis of the dependence of the electrical conductivity of liquid crystals on cell thickness is still missing. In this paper, we discuss how interactions between ions and substrates of the liquid crystal cell make their DC electrical conductivity dependent on the cell thickness.

\section{Model}

In the case of molecular thermotropic liquid crystals, their finite $D C$ electrical conductivity $\lambda_{D C}$ is caused by ions. It can be written as (1):

$$
\lambda_{D C}=\sum_{i} q_{i} \mu_{i} n_{i}
$$

where $q_{i}$ is the charge of the $i$-th ion, $\mu_{i}$ is the mobility of the $i$-th ion, and $n_{i}$ is its volume concentration [16,17]. In the case of two types of symmetric monovalent ions $\left(q_{i}=|e|(i=1,2)\right.$, $n_{1}^{+}=n_{1}^{-}=n_{1}, \mu_{1}=\mu_{1}^{+}+\mu_{1}^{-}, n_{2}^{+}=n_{2}^{-}=n_{2}, \mu_{2}=\mu_{2}^{+}+\mu_{2}^{-}$) the electrical conductivity can be rewritten as (2)

$$
\lambda_{D C}=|e|\left(\mu_{1} n_{1}+\mu_{2} n_{2}\right)
$$

where $|e|=1.6 \times 10^{-19} \mathrm{C}$.

The bulk concentration of ions can be found by applying a recently developed model [26,27]. This model considers the possibility of both ion capturing and ion releasing regimes in liquid crystal cells. The ion releasing regime takes place if substrates of a liquid crystal cell are contaminated with ions prior to filling the cell with liquid crystals. In this case, contaminated substrates act as sources of ion generation in liquid crystals. The trapping of ions by the substrates (in the simplest case via physical or chemical adsorption) results in the ion capturing regime. The processes of ion capturing and ion generation are described by rate Equation (3):

$$
\frac{d n_{j}}{d t}=-k_{S j}^{a \pm} n_{j} \frac{\sigma_{S j}}{d}\left(1-\Theta_{S 1}^{ \pm}-\Theta_{S 2}^{ \pm}\right)+k_{S j}^{d \pm} \frac{\sigma_{S j}}{d} \Theta_{S j}^{ \pm}
$$

where $j$ denotes the dominant type of symmetric ions in liquid crystals $(j=1,2), k_{S j}^{a \pm}$ is the effective rate constant corresponding to the ion capturing process of $n_{j}^{+}$and $n_{j}^{-}$ions on the surface of substrates, and $k_{S j}^{d \pm}$ is the effective rate constant characterizing the ion releasing process of $n_{j}^{+}$and $n_{j}^{-}$ions from the substrates, and $\Theta_{S j}^{ \pm}$is the fractional surface coverage of substrates corresponding to the $j$-th ions, $\sigma_{S j}$ is the surface density of all surface sites of the liquid crystal substrates, $n_{j}$ is the concentration (volume density) of mobile ions of the $j$-th type $(j=1,2), d$ is the thickness of the cell $[26,27]$. 
Equation (4) represents the conservation of the total number of ions of the $j$-th type:

$$
n_{0 j}+\frac{\sigma_{S j}}{d} v_{S j}=n_{j}+\frac{\sigma_{S j}}{d} \Theta_{S j}^{ \pm}
$$

where $v_{S j}$ is the contamination factor of substrates [26,27]. Recent papers [26,27] discussed the applicability and limits of the model described by Equations (3) and (4). Equations (3) and (4) are very general and can be applied to a wide range of molecular liquid crystals and substrates. The strength of interactions between ions and substrates determines the value of the parameter $K_{i}=\frac{k_{S i}^{a}}{k_{S i}^{d}}$, the size of ionic contaminants affects the value of the parameter $\sigma_{S i}$, and the level of ionic contamination of substrates is quantified by the contamination factor $v_{S i}$. The quantification of the ionic contamination of substrates is an important aspect of the proposed model. Depending on the type of materials (liquid crystals and substrates) and ionic contaminants (organic ions, inorganic ions), typical values of basic physical parameters used in this model $\left(K_{i}=\frac{k_{S i}^{a}}{k_{S i}^{d}}, \sigma_{S i}, v_{S i}, \mu_{i}\right)$ can vary over a wide range of values $\left(K_{i}=10^{-25}-10^{-20} \mathrm{~m}^{3}, \sigma_{S i}=10^{16}-10^{19} \mathrm{~m}^{-2}, v_{S i}=0-10^{-1}\right.$, additional information can be found in paper [26] and recent review [18]). In the present paper, the values of physical parameters were chosen to represent typical liquid crystal cells, such as cyanobiphenyl nematic liquid crystals sandwiched between polyimide alignment layers.

\section{Results}

DC electrical conductivity of liquid crystal cells as a function of their thickness was computed using Equations (2)-(4). Table 1 lists values of basic physical parameters used to generate graphs shown in Figures 1 and 2. As a rule, electrical measurements of liquid crystals are carried out using liquid crystal cells. The presence of substrates can lead to several ionic processes. The capturing of ions by substrates results in the ion capturing regime, whereas the use of contaminated substrates leads to the ion releasing regime. Normally, ions that are already present in the liquid crystal bulk prior to filling an empty cell get captured by the substrates once the cell is filled. This scenario is shown in Figures 1a and 2a. At the same time, if substrates of the empty cell are contaminated with ions, these ions contaminate liquid crystal materials upon filling the cell, as can be seen in Figures $1 \mathrm{~b}$ and $2 \mathrm{~b}$. The combination of these two, ion-releasing and ion-capturing, processes can result in a non-trivial dependence of the electrical conductivity on the cell thickness (Figures 1c and 2c).

Table 1. Values of physical parameters used to model DC electrical conductivity.

\begin{tabular}{cc}
\hline Physical Parameter & Value \\
\hline$K_{1}=\frac{k_{S 1}^{a}}{k_{S 1}^{d}}$ & $10^{-21} \mathrm{~m}^{3}$ (Figures 1 and 3a) \\
$K_{2}=\frac{k_{S 2}^{a}}{k_{S 2}^{d}}$ & $10^{-22} \mathrm{~m}^{3}$ (Figures 1 and 3a) \\
$K_{3}=\frac{k_{S 3}^{d}}{k_{s 3}^{d}}$ & $10^{-23} \mathrm{~m}^{3}$ (Figures 2 and 3b) \\
$K_{4}=\frac{k_{s 4}^{d}}{k_{S 4}^{d}}$ & $10^{-21} \mathrm{~m}^{3}$ (Figures 2 and 3b) \\
$\sigma_{S 1}=\sigma_{S 2}$ & $5 \times 10^{16} \mathrm{~m}^{-2}$ \\
$\mu_{1}=\mu_{2}$ & $10^{-10} \mathrm{~m}^{2} / \mathrm{Vs}$ \\
$v_{S 1}$ & 0 \\
$v_{S 2}$ & $10^{-3}($ Figures 1 and 2$)$ \\
$n_{01}$ & $10^{-2}$ (Figure 3a) \\
$n_{02}$ & $6 \times 10^{-4}$ Figure $\left.3 \mathrm{~b}\right)^{-3}$ \\
\hline
\end{tabular}



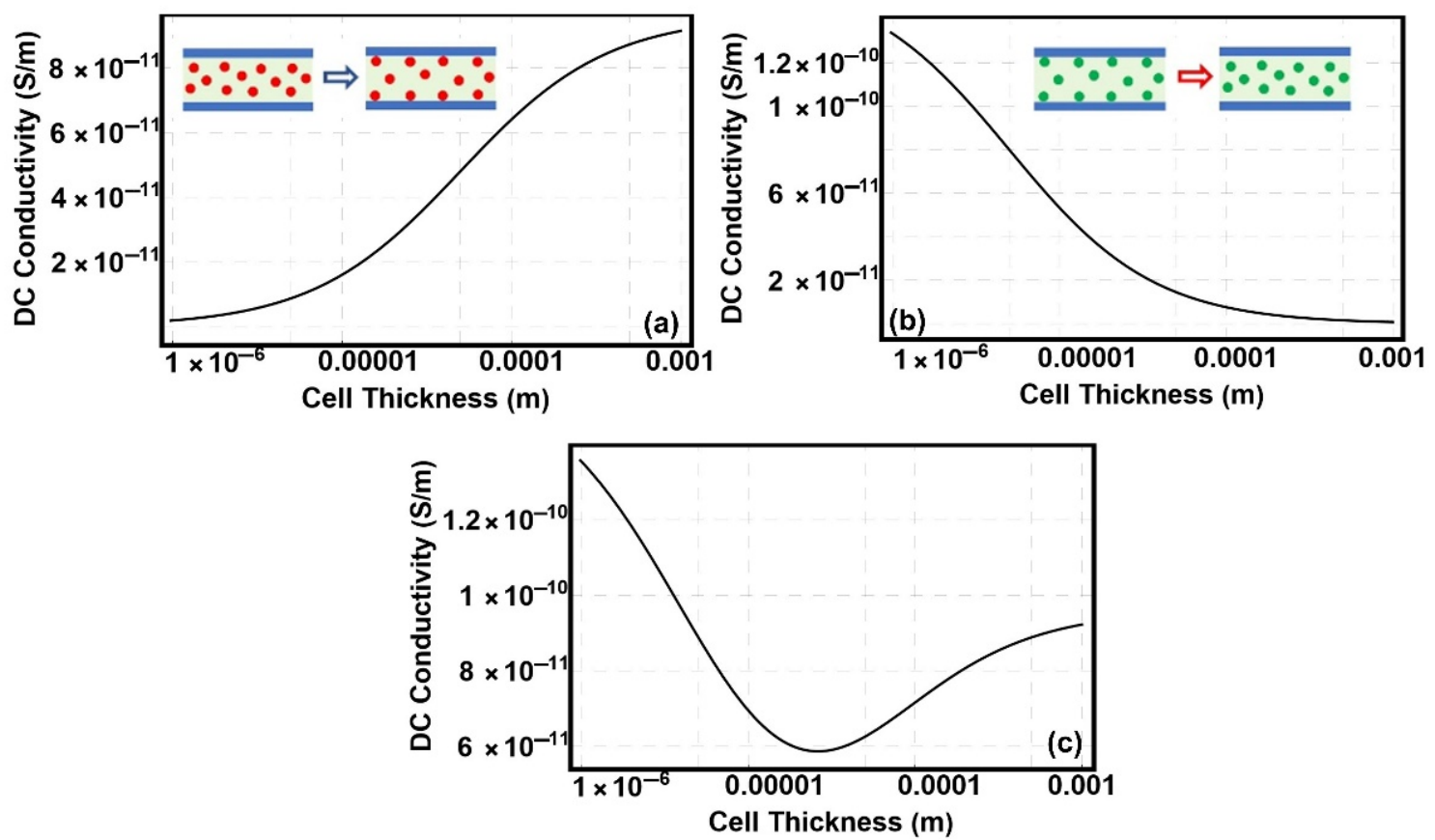

Figure 1. DC conductivity of liquid crystals as a function of the cell thickness. (a) DC conductivity caused by ions already present in liquid crystals. Values of parameters used in simulations are listed in Table $1\left(n_{01}, v_{S 1}, \mu_{1}, \sigma_{S 1}, K_{1}\right)$. (b) DC conductivity due to ions originated from contaminated substrates of the liquid crystal cell. Values of parameters used in simulations are listed in Table $1\left(n_{02}, v_{S 2}, \mu_{2}, \sigma_{S 2}, K_{2}\right)$. (c) DC conductivity caused by the combination of ion-capturing and ion-releasing processes shown in Figure 1a,b. Values of parameters used in simulations are listed in Table $1\left(n_{01}, n_{02}, v_{S 1}\right.$, $\left.v_{S 2}, \mu_{1}=\mu_{2}, \sigma_{S 1}=\sigma_{S 2}, K_{1}, K_{2}\right)$.
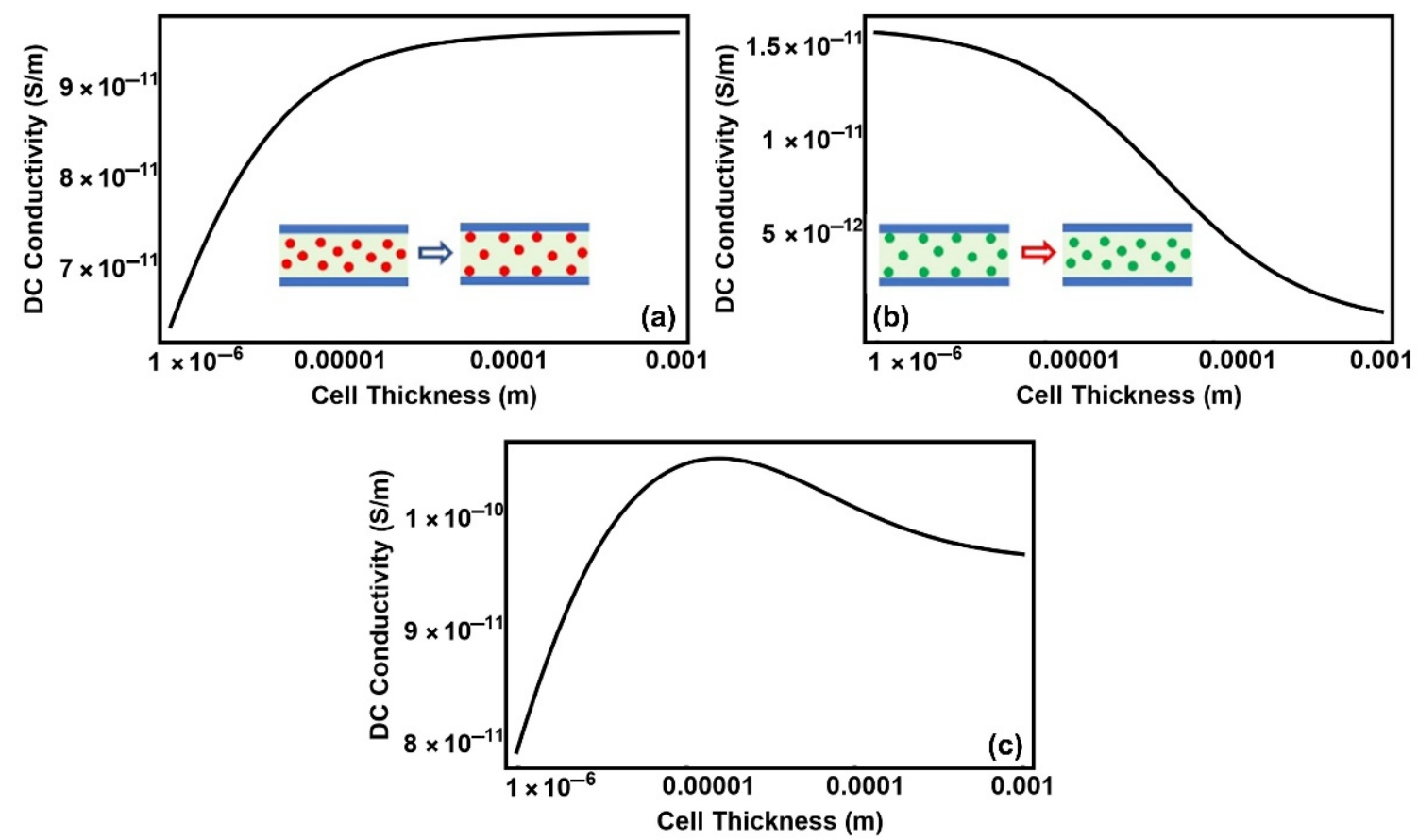

Figure 2. DC conductivity of liquid crystals as a function of the cell thickness. (a) DC conductivity caused by ions already present in liquid crystals. Values of parameters used in simulations are listed in Table $1\left(n_{01}, v_{S 1}, \mu_{1}, \sigma_{S 1}, K_{3}\right)$. (b) DC conductivity due to ions originated from contaminated substrates of the liquid crystal cell. Values of parameters used in simulations are listed in Table $1\left(n_{02}, v_{S 2}, \mu_{2}, \sigma_{S 2}, K_{4}\right)$. (c) DC conductivity caused by the combination of ion-capturing and ion-releasing processes shown in Figure 1a,b. Values of parameters used in simulations are listed in Table $1\left(n_{01}, n_{02}, v_{S 1}\right.$, $\left.v_{S 2}, \mu_{1}=\mu_{2}, \sigma_{S 1}=\sigma_{S 2}, K_{3}, K_{4}\right)$. 

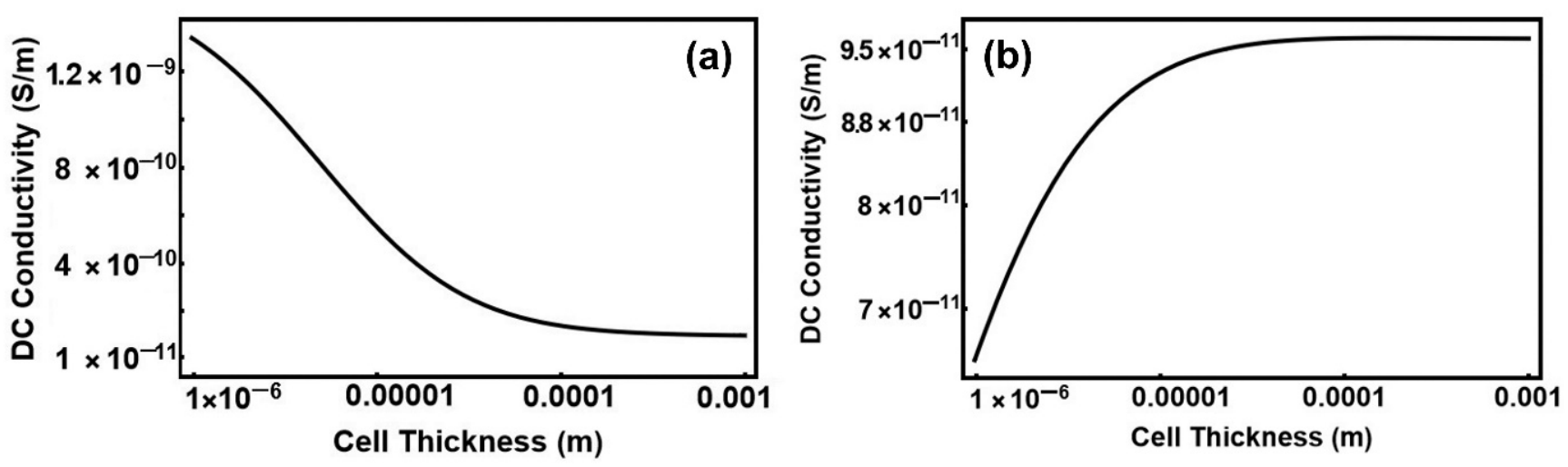

Figure 3. (a) Monotonous dependence of DC conductivity on the cell thickness achieved by increasing the value of the contamination factor $v_{S 2}$ by one order of magnitude (Table 1). (b) Monotonous dependence of DC conductivity on the cell thickness achieved by decreasing the value of the contamination factor $v_{S 2}$ by one order of magnitude (Table 1).

Interactions between ions and substrates of liquid crystal cells result in the dependence of DC electrical conductivity on the cell thickness. In general, this dependence can be monotonous or non-monotonous. Because monotonous dependence of DC electrical conductivity on the cell thickens is a rather trivial outcome, in the present paper, we decided to discuss some non-trivial cases corresponding to non-monotonous dependencies shown in Figures 1 and 2. At the same time, it is important to show other types of dependencies. For a given set of materials (ionic contaminants, liquid crystals, and substrates), the transition from non-monotonous dependence to monotonous one can be achieved by varying the contamination factor as shown in Figure 3a,b.

\section{Conclusions}

The dependence of DC electrical conductivity on cell thickness shown in Figures 1-3 has important practical implications. It suggests an importance to consider interactions between ions and substrates of the liquid crystal cell. These interactions can result in a combination of ion capturing and ion releasing effects. A striking manifestation of this interplay between ionic processes is a non-monotonous dependence of the conductivity on the cell thickness exhibiting either minimum (Figure 1c) or maximum (Figure 2c). It should be noted that ionic processes caused by the presence of substrates get weaker and become almost negligible if very thick $(>100 \mu \mathrm{m})$ cells are used (Figures 1-3). Because typical cells used in experiments are normally much thinner $(1-20 \mu \mathrm{m})$, the consideration of the dependence of DC electrical conductivity on the cell thickness is very important and should not be ignored. Electrical measurements of this type allow for the correct evaluation of the true values of the electrical parameters of liquid crystal materials. Moreover, they can also reveal possible sources of ionic contamination of substrates of liquid crystal cells.

Author Contributions: Conceptualization, Y.G.; methodology, Y.G.; formal analysis, D.W. and Y.G.; investigation, D.W. and Y.G.; data generation, D.W.; writing-original draft preparation, D.W. and Y.G.; writing-review and editing, D.W. and Y.G.; supervision, Y.G.; project administration, Y.G.; funding acquisition, Y.G. All authors have read and agreed to the published version of the manuscript.

Funding: This research was supported by the CSU-AAUP Faculty Research Grant and by the Faculty-Student Research Grant.

Data Availability Statement: All data that support the findings of this study are included within the article.

Acknowledgments: The authors would like to acknowledge the support provided by the School of Engineering, Science and Technology at Central Connecticut State University.

Conflicts of Interest: The authors declare no conflict of interest. 


\section{References}

1. Huang, Y.; Liao, E.; Chen, R.; Wu, S.-T. Liquid-Crystal-on-Silicon for Augmented Reality Displays. Appl. Sci. $2018,8,2366$. [CrossRef]

2. Otón, J.M.; Otón, E.; Quintana, X.; Geday, M.A. Liquid-crystal phase-only devices. J. Mol. Liq. 2018, 267, 469-483. [CrossRef]

3. Lazarev, G.; Chen, P.-J.; Strauss, J.; Fontaine, N.; Forbes, A. Beyond the display: Phase-only liquid crystal on Silicon devices and their applications in photonics. Opt. Express 2019, 27, 16206-16249. [CrossRef] [PubMed]

4. Abdulhalim, I. Non-display bio-optic applications of liquid crystals. Liq. Cryst. Today 2011, 20, 44-60. [CrossRef]

5. Lin, Y.; Wang, Y.; Reshetnyak, V. Liquid crystal lenses with tunable focal length. Liq. Cryst. Rev. 2017, 5, 111-143. [CrossRef]

6. De Sio, L.; Roberts, D.E.; Liao, Z.; Hwang, J.; Tabiryan, N.; Steeves, D.M.; Kimball, B.R. Beam shaping diffractive wave plates. Appl. Opt. 2018, 57, A118-A121. [CrossRef]

7. Chigrinov, V.G. Liquid Crystal Photonics; Nova Science Pub Inc.: New York, NY, USA, 2014; 204p.

8. Jeng, S.C. Applications of Tamm plasmon-liquid crystal devices. Liq. Cryst. 2020, 47, 1223-1231. [CrossRef]

9. Lininger, A.; Zhu, A.Y.; Park, J.S.; Palermo, G.; Chatterjee, S.; Boyd, J.; Capasso, F.; Strangi, G. Optical properties of metasurfaces infiltrated with liquid crystals. Proc. Natl. Acad. Sci. USA 2020, 117, 20390-20396. [CrossRef]

10. Geis, M.W.; Bos, P.J.; Liberman, V.; Rothschild, M. Broadband optical switch based on liquid crystal dynamic scattering. Opt. Express 2016, 24, 13812-13823. [CrossRef]

11. Konshina, E.A.; Shcherbinin, D.P. Study of dynamic light scattering in nematic liquid crystal and its optical, electrical and switching characteristics. Liq Cryst. 2018, 45, 292-302. [CrossRef]

12. Shaban, H.; Wu, P.-C.; Lee, J.-H.; Lee, W. Dielectric and electro-optical responses of a dielectrically negative nematic liquid crystal doped with cationic surfactant. Opt. Mater. Express 2021, 11, 3208-3222. [CrossRef]

13. Dabrowski, R.; Dziaduszek, J.; Bozetka, J.; Piecek, W.; Mazur, R.; Chrunik, M.; Perkowski, P.; Mrukiewicz, M.; Żurowska, M.; Weglowska, D. Fluorinated smectics-New liquid crystalline medium for smart windows and memory displays. J. Mol. Liq. 2017, 267, 415-427. [CrossRef]

14. Zhang, Y.; Yang, X.; Zhan, Y.; Zhang, Y.; He, J.; Lv, P.; Yuan, D.; Hu, X.; Liu, D.; Broer, D.J.; et al. Electroconvection in zwitterion-doped nematic liquid crystals and application as smart windows. Adv. Opt. Mater. 2020, 27, 2001465. [CrossRef]

15. Camley, R.; Celinski, Z.; Garbovskiy, Y.; Glushchenko, A. Liquid crystals for signal processing applications in the microwave and millimeter wave frequency ranges. Liq. Cryst. Rev. 2018, 6, 17-52. [CrossRef]

16. Blinov, L.M. Structure and Properties of Liquid Crystals; Springer: New York, NY, USA, 2010.

17. Neyts, K.; Beunis, F. Ion Transport in Liquid Crystals. In Handbook of Liquid Crystals: Physical Properties and Phase Behavior of Liquid Crystals; Wiley-VCH: Weinheim, Germany, 2014; Volume 2, Chapter 11; pp. 357-382.

18. Garbovskiy, Y. Conventional and unconventional ionic phenomena in tunable soft materials made of liquid crystals and nanoparticles. Nano Ex. 2021, 2, 012004. [CrossRef]

19. Colpaert, C.; Maximus, B.; Meyere, D. Adequate measuring techniques for ions in liquid crystal layers. Liq. Cryst. 1996, 21, 133-142. [CrossRef]

20. Barbero, G.; Evangelista, L.R. Adsorption Phenomena and Anchoring Energy in Nematic Liquid Crystals; Taylor \& Francis: Boca Raton, FL, USA, 2006.

21. Khazimullin, M.V.; Lebedev, Y.A. Influence of dielectric layers on estimates of diffusion coefficients and concentrations of ions from impedance spectroscopy. Phys. Rev. E 2019, 100, 062601. [CrossRef]

22. Karaawi, A.R.; Gavrilyak, M.V.; Boronin, V.A.; Gavrilyak, A.M.; Kazachonok, J.V.; Podgornov, F.V. Direct current electric conductivity of ferroelectric liquid crystals-gold nanoparticles dispersion measured with capacitive current technique. Liq. Cryst. 2020, 47, 1507-1515. [CrossRef]

23. Garbovskiy, Y. Evaluating the Concentration of Ions in Liquid Crystal Cells: Hidden Factors and Useful Techniques. Proceedings 2020, 62, 10. [CrossRef]

24. Dhara, S.; Madhusudana, N.V. Ionic contribution to the dielectric properties of a nematic liquid crystal in thin cells. J. Appl. Phys. 2001, 90, 3483-3488. [CrossRef]

25. Kumar, A.; Varshney, D.; Prakash, J. Role of ionic contribution in dielectric behaviour of a nematic liquid crystal with variable cell thickness. J. Mol. Liq. 2020, 303, 112520. [CrossRef]

26. Garbovskiy, Y. Ion capturing/ion releasing films and nanoparticles in liquid crystal devices. Appl. Phys. Lett. 2017, 110, 041103. [CrossRef]

27. Garbovskiy, Y. Ions and size effects in nanoparticle/liquid crystal colloids sandwiched between two substrates. The case of two types of fully ionized species. Chem. Phys. Lett. 2017, 679, 77-85. [CrossRef] 